\title{
Relación entre las complicaciones y la calidad de vida del paciente en hemodiálisis
}

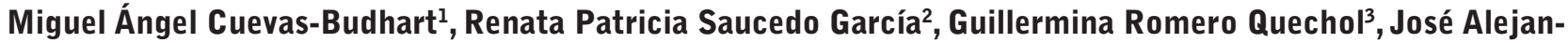 \\ dro García Larumbe ${ }^{4}$, Anwar Hernández Paz y Puente ${ }^{5}$
}

Hospital General Regional N 1 "Vicente Guerrero". Acapulco. Guerrero. México, Unidad de Investigación Médica de Enfermedades Endocrinas. Hospital de Especialidades. CMN Siglo XXI, Unidad de Investigación Epidemiológica y en Servicios de Salud. IMSS. Ciudad de México. México.

\section{Resumen}

Introducción: Los pacientes con Enfermedad Renal Crónica (ERC) son tratados con terapias de diálisis. Dentro de este tipo de tratamiento se encuentran la Diálisis Peritoneal (DP) y Hemodiálisis (HD). Los pacientes sometidos a HD tienen una evolución imprevisible por las complicaciones del tratamiento y/o complicaciones propias de la ERC. Estas aumentan el número de hospitalizaciones y deterioran la calidad de vida (CV).

Objetivos: Evaluar la calidad de vida de pacientes en hemodiálisis y determinar la asociación entre las complicaciones y la CV.

Material y Método: Estudio transversal analítico en 157 pacientes en HD (75 hombres, 82 mujeres), mayores de 18 años y con más de 3 meses en tratamiento. La CV se evaluó con el instrumento KDQOL-36, el cual, mide 5 dimensiones en escala del 0 al 100. Se realizó un análisis bivariado, ANOVA y regresión múltiple para evaluar la relación de cada una de las dimensiones con edad, sexo, ocupación, estado civil, escolaridad, tipo de acceso venoso, tiempo con la ERC, con la HD y complicaciones de la ERC y la HD.

Resultados: La edad promedio fue de 50.9 años. El $77 \%$ de los participantes presentaron complicaciones, $69.4 \%$ por HD, $5 \%$ por evolución de la ERC y $25.6 \%$ ambas complicaciones. En el análisis multivariado se encontró que la presencia de ambas complicaciones

Correspondencia:
Miguel Ángel Cuevas Budhart
Infonavit alta progreso. Edif. 37 depto. $4024^{\text {ta }}$ etapa
39610 Acapulco Guerrero. México
E-mail: angel_budhart@hotmail.com

deteriora más la calidad de vida que las ocasionadas únicamente por el tratamiento de HD.

Conclusión: Las complicaciones del tratamiento de hemodiálisis aunadas a las de la ERC deterioran en gran medida la calidad de vida del paciente.

PALABRAS CLAVE: calidad de vida; hemodiálisis; complicaciones.

Relationship between complications and the quality of life of the patient on hemodialysis

\section{Summary}

Introduction: Patients with Cronic Kidney Disease (CKD) are treated with dialysis therapies. Within this type of treatment are Peritoneal Dialysis (PD) and Hemodialysis (HD). Patients undergoing HD have unexpected turn for treatment complications and / or complications of CKD. These increase the number of hospitalizations and deteriorate the quality of life (QOL).

Objective: Evaluate the Quality of Life of Patients on hemodialysis and determine the association between complications and QoL.

Material and Method: Analytical cross-sectional study in 157 patients in HD (75 men, 82 women), aged 18 years and over 3 months treatment. QoL was assessed with the KDQOL-36 instrument, which, measuring 5 dimensions on a scale of 0 to 100 . Was conducted of the bivariate analysis, ANOVA and and multiple regression to assess the ratio $v$ Each Dimensions with 
Age, sex, occupancy, marital status, education, access type venous access time with the ERC, with HD and complications of CKD and HD.

Results: Average age was $50.9 \mathrm{~m}$ years. $77 \%$ of participants had complications, $69.4 \%$ for HD, 5\% Evolution of CKD and 25.6\% Complications Both. In the multivariate analysis it was found that the presence of both complications deteriorates the complications caused only by the HD tratment.

Results: Average age was $50.9 \mathrm{~m}$ years. $77 \%$ of participants had complications, $69.4 \%$ for HD, 5\% Evolution of CKD and $25.6 \%$ Complications Both. In the multivariate analysis it was found that the presence of both complications deteriorates the complications caused only by the HD tratment.

Conclusion: Hemodialysis tratment complication together with CKD complications greatly deteriorate patinents quality of life.

KEYWORDS: quality of life; hemodialysis; complications.

\section{Introducción}

La Enfermedad Renal Crónica (ERC) es el deterioro progresivo e irreversible de la función renal con una duración igual o mayor a 3 meses, cuyo grado de afectación se determina con un filtrado glomerular (FG) $<60 \mathrm{ml} / \mathrm{min} / 1.73 \mathrm{~m}^{2}$. Los riñones pierden su capacidad de eliminar desechos del organismo, concentrar orina y conservar los electrolitos en la sangre ${ }^{1}$. La Diabetes Mellitus tipo 2 (DM2) y la hipertensión arterial sistemática, son los principales factores etiológicos de la ERC². En México es un grave problema de salud pública ya que se encuentra dentro de las 10 principales causas de muerte. Este padecimiento no solo es un asunto importante por su magnitud, si no también porque se presenta en edades tempranas. Actualmente se calcula una incidencia de 377 casos por millón de habitantes y una prevalencia de 1,142 por millón de habitantes ${ }^{3}$.

Los pacientes con ERC son tratados con terapias de sustitución renal como la Diálisis Peritoneal (DP), Hemodiálisis (HD) y Trasplante Renal ${ }^{4}$. La HD es un procedimiento altamente invasivo, en el que se emplean dializadores con membranas artificiales (celulósica sintética). Los pacientes sometidos a este tipo de tratamiento se encuentran en riesgo de desarrollar algún tipo de complicación a causa del tratamiento, observándose cambios físicos, químicos, hidroelectrolíticos, ácido-básicos, y hemodinámicos. La presencia de complicaciones por HD aumenta la demanda de hospitalización generando altos costos para las instituciones de salud, al paciente y a su familia, afectando el aspecto físico, psicológico y social, lo cual puede repercutir en su calidad de vida (CV) $)^{5-7}$.

El término calidad de vida se describió en 1975 y se expresó en términos de bienestar, como un proceso dinámico y cambiante, que varía según el sistema de valores de los individuos. En 1994, la Organización Mundial de la Salud (OMS) creó el grupo World Health Organization Quality of Life (WHOQOL) para medir la CV quien la definió como: "La percepción que un individuo tiene de su lugar en la existencia, en el contexto de la cultura y del sistema de valores en los que vive y en relación con sus objetivos, sus expectativas, sus normas, sus inquietudes". Desde entonces se ha convertido en un indicador de calidad de los tratamientos proporcionados por el sistema de salud ${ }^{8-10}$.

La CV se evalúa frecuentemente en pacientes con ERC a través de instrumentos genéricos como el SF36 creado en los Estados Unidos a principios de los noventa, sin embargo, cuando existe una pérdida de salud como en los pacientes con Tratamiento Renal Sustitutivo (TRS) no es el instrumento apropiado. Para la evaluación de la CV en pacientes con ERC el cuestionario más idóneo es el The Kidney Disease Quality of Life (KDQOL) ya que es un instrumento de medición específico y genérico que cuenta con 36 ítems y fue diseñado para pacientes con ERC que se encuentren bajo TRS. Este instrumento ha sido validado y usado ampliamente en estudios internacionales, mide 2 escalas de componente genérico salud física y salud mental, y 3 sub-escalas específicas que miden síntomas y problemas, carga de la enfermedad renal y efectos de la enfermedad renal. En un estudio realizado en Chile se le determinó un Alfa de Cronbach $0.82,0.67$, y 0.76 para cada una de las sub-escalas mencionadas con antelación ${ }^{11}$. El cuestionario KDQOL 36 también ha sido validado en población mexicana y se ha utilizado en encuestas de CV en pacientes con TRS en el estado de 0axaca ${ }^{12}$.

El estado de Guerrero ocupa el tercer lugar a nivel nacional en Años de Vida Saludable (AVISA) perdidos por ERC, siendo la cuarta causa de muerte en el estado, antecediéndole la violencia, DM2 y cardiopatías isquémicas $^{13}$. Por ello la importancia de medir la frecuencia de complicaciones y eventos durante el tratamiento de 
hemodiálisis, así como la repercusión que estas tienen sobre la CV.

\section{Objetivo}

Evaluar la calidad de vida de pacientes en hemodiálisis y determinar la asociación entre las complicaciones y la CV.

\section{Material y Método}

Se realizó un estudio transversal analítico multicéntrico en el periodo comprendido de febrero a junio del 2016 en el estado de Guerrero, evaluando 5 unidades privadas y 2 públicas. El muestreo fue no probabilístico por conveniencia. Se estudiaron 157 pacientes de ambos sexos, mayores de 18 años y en tratamiento de HD por más de 3 meses. Se obtuvo el consentimiento informado por escrito de los pacientes, explicándoles el objetivo del estudio y el riesgo mínimo de participar en el mismo de acuerdo a las normas de Helsin$\mathrm{ki}^{14}$.

Se utilizó el cuestionario KDQ0L-36 que mide 5 dimensiones y permite obtener 2 puntuaciones generales del SF-36: componente de salud física y componente de salud mental. El componente específico del KDQ0L-36, permite obtener las puntuaciones de las sub-escalas específicas: síntomas y problemas, efectos de la enfermedad renal y carga de la enfermedad renal ${ }^{12}$. Al instrumento se le agregaron variables sociodemográficas, datos de laboratorio, evolución de la enfermedad, complicaciones propias de la enfermedad y del tratamiento de HD.

La recolección de información se utilizó con una técnica de entrevista cara a cara y se revisó el expediente clínico para corroborar la información proporcionada por el paciente. Se registró la etiología de la enfermedad, datos de laboratorio, complicaciones y frecuencia de hospitalizaciones. Para la captura de información del instrumento de medición de CV se utilizó el programa KDQ0L TM-36 Scoring Program (v 2.0) en Excel. Para el análisis estadístico se elaboró una base de datos en el programa IBM SPSS Statistics 22.

En la realización del análisis estadístico las variables categóricas se describieron como frecuencias simples y proporciones y las variables cuantitativas como promedio y desviación estándar $(\bar{X} \pm D S)$. Se realizó un análisis bivariado y dado que la variable dependiente se aproximó al modelo de curva normal, se aplicó ANOVA para evaluar diferencias de las 5 dimensiones de calidad de vida entre los distintos grupos de estudio, los cuales se clasificaron de la siguiente manera: sin complicaciones, complicaciones de la ERC, 1 a 2 complicaciones del tratamiento de HD, > 3 complicaciones de la HD y ambos tipos de complicaciones. También se utilizó ANOVA para evaluar diferencias en CV por estado civil, escolaridad y tipo de acceso venoso. Se realizó correlación de Pearson para analizar la asociación de edad y dimensiones de calidad de vida y la prueba $t$ Student para evaluar diferencias de $\mathrm{CV}$ en relación al sexo. Finalmente se realizó una regresión múltiple utilizando como variable dependiente cada una de las dimensiones de calidad de vida y como variables independientes la edad, sexo, ocupación, estado civil, escolaridad, tiempo con la ERC, tiempo con la HD, tipo de acceso venoso, etiología de la ERC, complicaciones de la HD, complicaciones de la ERC y ambas complicaciones. Se consideró significativo un valor de $\mathrm{P}<0.05$.

\section{Resultados}

Las principales características sociodemográficas se muestran en la tabla 1.

Tabla 1. Datos sociodemográficos, clínicos y etiología de la ERC.

\begin{tabular}{ll}
\hline $\begin{array}{l}\text { Edad (años) } \\
\text { Fexo }\end{array}$ & $50.92 \pm 14.7$ \\
\hline Masculino & $82(52.2)$ \\
\hline $\begin{array}{c}\text { Escolaridad } \\
\text { Primaria }\end{array}$ & $75(47.8)$ \\
\hline Secundaria & $77(49.0)$ \\
\hline Preparatoria & $30(19.1)$ \\
\hline Licenciatura & $26(16.6)$ \\
\hline Maestría & $22(14.0)$ \\
\hline \hline Ocupación & $2(1.3)$ \\
\hline Hogar & \\
\hline Comerciante & $64(40.8)$ \\
\hline Empleado & $16(10.2)$ \\
\hline Profesionista & $23(14.6)$ \\
\hline Pensionado & $5(3.2)$ \\
\hline No Trabaja & $17(10.8)$ \\
\hline
\end{tabular}




\begin{tabular}{|c|c|}
\hline \multicolumn{2}{|l|}{ Estado Civil } \\
\hline Soltero & $31(19.7)$ \\
\hline Casado & $101(64.3)$ \\
\hline Divorciado & $5(3.2)$ \\
\hline Unión Libre & $7(4.5)$ \\
\hline Viudo & $13(8.3)$ \\
\hline \multicolumn{2}{|l|}{ Presión Arterial } \\
\hline Sistólica $(\mathrm{mm} / \mathrm{Hg})$ & $146.2 \pm 27.3$ \\
\hline Diastólica $(\mathrm{mm} / \mathrm{Hg})$ & $81.8 \pm 14.4$ \\
\hline \multicolumn{2}{|l|}{ Paraclínicos } \\
\hline Creatinina (mg/dl) & $7.5 \pm 3.7$ \\
\hline Glucosa (mg/dl) & $106.0 \pm 40.4$ \\
\hline Colesterol (mg/dl) & $158.3 \pm 46.6$ \\
\hline Triglicéridos (mg/dl) & $166.5 \pm 79.5$ \\
\hline Hemoglobina ( $\mathrm{g} / \mathrm{dl})$ & $12.8 \pm 14.3$ \\
\hline Albumina $(\mathrm{mg} / \mathrm{dl})$ & $4.1 \pm 2.2$ \\
\hline \multicolumn{2}{|l|}{ Etiología } \\
\hline Diabetes Mellitus Tipo 2 & $50(31.8)$ \\
\hline Hipertensión Arterial & $33(21.0)$ \\
\hline Poliquistosis Renal & $9(5.7)$ \\
\hline Litiasis Renal & $7(4.5)$ \\
\hline Pielonefritis & $4(2.5)$ \\
\hline \multicolumn{2}{|l|}{ Nefroangioesclerosis 9 (5.7) } \\
\hline Uropatia Obstructiva Crónica & $5(3.2)$ \\
\hline Origen Desconocido & $10(6.4)$ \\
\hline Hipoplasia Renal & $6(3.8)$ \\
\hline${ }^{*}$ Otros & $24(15.3)$ \\
\hline
\end{tabular}

*Eclampsia, LES, Glomerulopatías, falla y rechazo de trasplante renal Los datos cuantitativos se expresan en X+DE y los cualitativos con n (\%)

El promedio de edad de los 157 participantes fue de 50.9 años, la frecuencia de hombres y mujeres evaluados fue similar. La mitad de los pacientes tienen como nivel máximo de estudios la primaria. Los principales factores etiológicos de la ERC fueron diabetes mellitus tipo 2 e hipertensión arterial.

El $77.1 \%$ de los pacientes presentó algún tipo de complicación, el $69.4 \%$ presento complicaciones por la HD, el $5 \%$ por la ERC y el $25.6 \%$ presentó ambas complicaciones.

Las complicaciones presentadas por la HD fueron: hipotensión (35.5\%) infección de angioacceso $(24.0 \%)$, cefalea $(22.3 \%)$, crisis hipertensiva $(14.5 \%)$, ma- reos $(9.9 \%)$, escalofríos $(9.9 \%)$, y trombosis $(9.9 \%)$. La estenosis, aneurisma de fístula, dolor precordial y convulsiones se presentaron con una frecuencia de $0.8 \%$.

Las complicaciones por la evolución de la ERC fueron: anemia (41.5\%), hiperparatiroidismo secundario $(15.1 \%)$, alteraciones electrolíticas $(7.5 \%)$, osteodistrofias (11.3\%), espondiloatropatías (5.7\%). La acidosis metabólica, poliglobulia y litiasis renal se presentaron con una frecuencia de $1.9 \%$.

Al evaluar la calidad de vida en todos los pacientes se encontró que la dimensión menos afectada fue síntomas y problemas con 72.6 y la de mayor afectación fue carga de la enfermedad con 36.5 (figura 1).

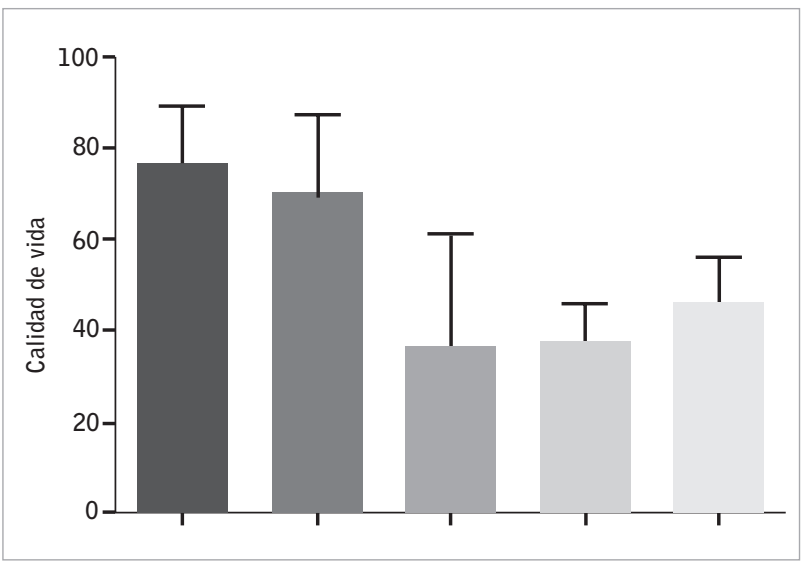

Figura 1. Calidad de vida global por dimensiones.

Al comparar la calidad de vida entre aquellos pacientes sin complicaciones, los pacientes con complicaciones por la ERC, los que presentaron de 1 a 2 complicaciones por HD, los de $>3$ complicaciones por HD y los de ambas complicaciones, se encontró que para la dimensión de síntomas y problemas los grupos de 1 a 2 complicaciones por HD, $>3$ complicaciones por HD, y los que tienen ambas complicaciones tienen menor calidad de vida que los que no han presentado ningún tipo de complicación.

Para la dimensión de efectos de la enfermedad renal el grupo afectado fue el de $>3$ complicaciones por HD. Para la dimensión de carga de la enfermedad renal el grupo que se vio afectado fue de 1 a 2 complicaciones de la HD, $>3$ complicaciones por HD y de ambas. Para los de la dimensión de componente de salud física se vio más afectado los de 1 a 2 complicaciones de la HD y ambas complicaciones, y finalmente en la dimensión de componente de salud mental los 
grupos más afectados fueron los que han tenido complicaciones de la HD de 1 a 2 y mayor de 3 , al igual que los que tienen ambas complicaciones. (figura 2 ).

En el análisis de regresión múltiple se encontró que la presencia de ambas complicaciones se asocia con las dimensiones de síntomas y problemas $(B=0.38 ; P=0.001)$, carga de la ERC $(\beta=0.41$; $\mathrm{P}=<0.001)$, componente físico ( $B=0.30 ; P=0.010$ ), y componente mental $(B=0.41 ; \quad P<0.001)$. Por otra parte, las complicaciones por HD se asociaron con síntomas y problemas ( $B=0.34 ; P=0.005)$, y carga de la enfermedad $(B=0.30$; $\mathrm{P}=0.021$ ). Finalmente, la complicación por la ERC se asocia únicamente con efectos de la ERC ( $B=0.30$; $\mathrm{P}=0.003$ ).

Al correlacionar edad y calidad de vida se encontró asociación negativa entre edad y síntomas y problemas $(r=-0.296, P<0.001)$, carga de la enfermedad renal $(r=-0.172, P=-0.031)$, componente de salud física $(r=$

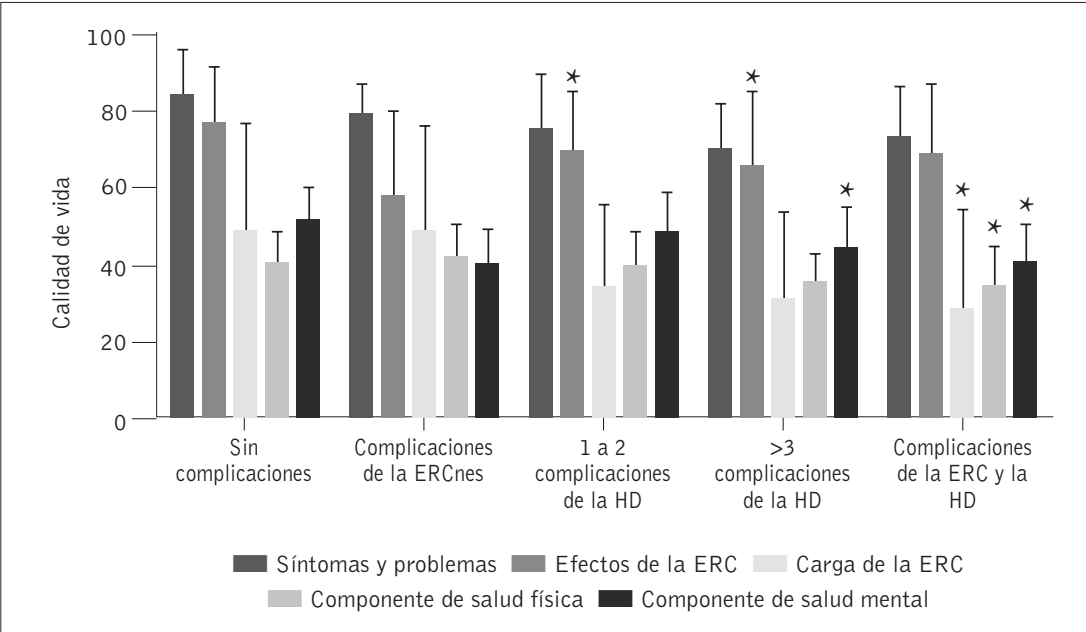

Figura 2. Comparación de las medias de las dimensiones entre grupos de estudio. * Diferencia estadística en comparación con las dimensiones del grupo sin complicaciones.

Tabla 2. Relación ente sexo, estado civil y escolaridad con calidad de vida.

\begin{tabular}{|c|c|c|c|c|c|}
\hline & $\begin{array}{l}\text { Lista de síntomas/ } \\
\text { problemas }\end{array}$ & $\begin{array}{l}\text { Efectos de la } \\
\text { enfermedad renal }\end{array}$ & $\begin{array}{l}\text { Carga de la } \\
\text { enfermedad renal }\end{array}$ & $\begin{array}{l}\text { Componente de } \\
\text { salud física }\end{array}$ & $\begin{array}{l}\text { Componente de } \\
\text { salud Mental }\end{array}$ \\
\hline $\begin{array}{l}\text { Sexo } \\
\text { Femenino }\end{array}$ & $74.5 \pm 13.5$ & $68.7 \pm 16.5$ & $33.8 \pm 25.2$ & $38.5 \pm 8.8$ & $45.0 \pm 10.1$ \\
\hline Masculino & $77.9 \pm 12.9$ & $71.3 \pm 18.0$ & $39.3 \pm 24.8$ & $37.3 \pm 8.4$ & $48.0 \pm 10.3$ \\
\hline $\begin{array}{l}\text { Estado civil } \\
\text { Soltero }\end{array}$ & $78.1 \pm 14.1$ & $68.4 \pm 17.6$ & $42.1 \pm 24.2$ & $39.7 \pm 9.34$ & $49.6 \pm 10.5$ \\
\hline Casado & $75.8 \pm 12.4$ & $70.6 \pm 17.2$ & $36.3 \pm 25.5$ & $37.6 \pm 8.4$ & $46.3 \pm 10.0$ \\
\hline Divorciado & $70.4 \pm 10.1$ & $66.3 \pm 13.1$ & $26.3 \pm 18.4$ & $35.5 \pm 8.1$ & $47.5 \pm 7.7$ \\
\hline Unión libre & $83.9 \pm 17.1$ & $70.1 \pm 24.1$ & $35.7 \pm 33.2$ & $42.1 \pm 8.2$ & $42.8 \pm 11.6$ \\
\hline Viudo & $72.8 \pm 15.9$ & $70.8 \pm 15.5$ & $28.4 \pm 20.7$ & $34.8 \pm 8.0$ & $41.7 \pm 10.4$ \\
\hline $\begin{array}{l}\text { Escolaridad } \\
\text { Primaria }\end{array}$ & $75.1 \pm 14.1$ & $72.1 \pm 16.6$ & $35.6 \pm 24.0$ & $37.3 \pm 7.9$ & $45.9 \pm 9.8$ \\
\hline Secundaria & $77.8 \pm 10.2$ & $72.8 \pm 14.0$ & $33.5 \pm 20.0$ & $28.4 \pm 9.3$ & $48.4 \pm 9.2$ \\
\hline Preparatoria & $78.9 \pm 12.9$ & $66.6 \pm 20.3$ & $33.2 \pm 24.0$ & $38.8 \pm 9.2$ & $44.8 \pm 12.3$ \\
\hline Licenciatura & $74.1 \pm 14.9$ & $62.9 \pm 18.6$ & $44.6 \pm 33.9$ & $38.2 \pm 9.9$ & $46.8 \pm 10.5$ \\
\hline Maestría & $82.3 \pm 1.5$ & $68.8 \pm 8.8$ & $65.6 \pm 13.3$ & $39.5 \pm 5.8$ & $56.9 \pm 14.4$ \\
\hline
\end{tabular}

Valores expresados en $\bar{X} \pm D S$.

-0.226, $\mathrm{P}=0.004)$, componente de salud mental ( $r=$ $-0.180 \mathrm{P}=0.024)$. Sin embargo, no se encontró asociación entre edad y efectos de la enfermedad renal.

Al relacionar las variables independientes de sexo, estado civil y escolaridad no se encontró relación de estas con la calidad de vida (tabla 2). 
De acuerdo al tipo de acceso venoso se encontró diferencia significativa únicamente en la dimensión de carga de la enfermedad renal entre la fístula arterio-venosa y el catéter semipermanente (56.7 vs 50.9, $\mathrm{P}=0.007$ ).

En relación al tiempo que lleva con la hemodiálisis no se encontró una relación con ninguna de las 5 dimensiones, sin embargo, como se muestra en la figura 3 el tiempo que lleva con la ERC si se asoció de manera positiva con la carga de la enfermedad renal $(r=0.197$, $\mathrm{P}=0.013$ ).

\section{Discusión}

Los pacientes con ERC sometidos a HD y con complicaciones del tratamiento tienen una disminución de la CV a diferencia de las complicaciones propias de la evolución de la enfermedad, sin embargo, los pacientes que tienen ambos tipos de complicaciones su CV se ve aún más afectada.

En comparación con el estudio realizado en Chile por Zuñiga Carlos ${ }^{15}$ en 2009 en el que evaluó la CV en pacientes en hemodiálisis crónica utilizando el cuestionario KDQOL 36 obteniendo resultados bajos en el componente físico y carga de la enfermedad renal con un 38.17 y 28.82 respectivamente, en el que la media de edad fue de 55 años. Habiendo muy poca variabilidad a la encontrada en este estudio ya que el promedio de edad fue de 50.9 años. Sin embargo, existe una similitud en las dimensiones afectadas carga de la enfermedad renal 36.5 y componente de salud física 37.9, pero con una leve variedad en el resultado, encontrando también el componente de salud mental afectado, esto puede deberse a que el paciente que inicia en HD cursa por periodos de depresión, que se plantea en artículos sobre el tema, por lo cual nos encontramos ante la necesidad de incorporar a los pacientes en hemodiálisis a grupos de apoyo y psicología.

Por otro lado, los resultados de este estudio son similares a los encontrados por Alicia Sánchez García ${ }^{16}$ en el 2012 obteniendo el mismo espectro de complicaciones. Sin embargo, existe una discrepancia en el resultado porcentual ya que en su estudio encontró calambres e hipertensión las principales complicacio- nes con un $71.2 \%$ y $67.3 \%$ respectivamente, esto se debe a que su estudio se vio limitado a 52 pacientes a diferencia de este estudio que contó con 157. Cabe mencionar que hay una similitud en cuanto al tipo de etiología de la ERC siendo la Diabetes mellitus (DM) e Hipertensión principales protagonistas del deterioro de la función renal (ambos estudios realizados en población mexicana), esto puede deberse a que México ocupa el sexto lugar a nivel mundial en DM y solo se cuenta con una cuarta parte en control metabólico, por lo cual el resto puede sufrir complicaciones graves como la Enfermedad renal crónica.

En el estudio realizado por Fernández Samos $\mathrm{R}$ y cols $^{17}$ muestra que la edad influye en la calidad de vida atenuando el impacto de la Enfermedad renal crónica siendo los más ancianos los que presentan mejor puntuación en la calidad de vida que los más jóvenes, en comparación con este estudio encontramos una diferencia ya que a mayor edad existe una peor calidad de vida, esto se debe a que en propio envejecimiento es por sí mismo un proceso cuya calidad de vida se ve frecuentemente afectada en la forma como el individuo satisface sus necesidades, la seguridad de ingreso económico y el contar con el apoyo familiar.

En este estudio se encontró una diferencia significativa entre la Fistula Arteriovenosa (FAV) y el catéter semipermanente esto puede deberse a que el catéter "Permcath" se instala como último recurso, cuando se terminan los Accesos Venosos (AV) del paciente, no son aptos para FAV por lo cual esto se ve reflejado en la CV. Algunos estudios mencionan que el Catéter Venoso Central (CVC) para HD es una alternativa del AV permanente por lo cual debe retirarse tan pronto como cese su indicación clínica ya que las complica- 
ciones tras la implantación de un CVC para HD no son infrecuentes, por lo cual esto influye en la CV de los pacientes.

En el presente estudio se encontró una asociación positiva entre la CV en su dimensión carga de la enfermedad renal y años con la ERC. Esto puede deberse a que con el paso del tiempo el paciente acepta más su enfermedad 0 aprende a vivir con ella.

Algunos otros estudios han descrito que existe una asociación entre la calidad de vida con sexo, estado civil y escolaridad, sin embargo, los resultados de este estudio mostraron una asociación nula entre las variables mencionadas con antelación, y la CV. Esto puede deberse a que la calidad de vida no solo depende del conocimiento que no tenga o de la compañía con que se cuente, sino tambien a las condiciones de vida y trabajo laboral si se está en una edad reproductiva.

Una limitación de estudio fue la muestra de pacientes que asisten a HD en los municipios de Iguala, $\mathrm{Zi}$ huatanejo y Acapulco que representan región norte, costa grande y costa centro del estado, estas regiones representan gran parte del estado sin embargo para comparar y validar resultados entre municipios se requiere realizar un estudio similar en la región centro que corresponde a Chilpancingo.

Es posible afirmar que este estudio es de importancia para el profesional de enfermería ya que habiendo identificado las complicaciones principales nos permite individualizar el cuidado, prevenir complicaciones, y promover la salud en prevención de la enfermedad renal crónica.

En este sentido los resultados del estudio enfatizan la necesidad de contar con proyectos educativos dirigidos a los pacientes que inicien tratamiento de hemodiálisis con el propósito de prevenir la aparición de complicaciones, esto permitirá una mejor evolución de la enfermedad y por ende una mejor calidad de vida.

Al igual que proponer estudios de cohorte prospectivos en los pacientes que presentaron complicaciones basándose en el estudio de Pautas de Práctica y Resultados de Diálisis (DOPSS) utilizando como directrices; Kt/V, Hemoglobina, Albúmina, Fósforo, Calcio y uso de catéteres, para medir el riesgo de mortalidad relativa.

\section{Conclusión}

La calidad de vida se ve francamente afectada cuando se cursa con complicaciones tanto del tratamiento de HD como de la evolución de la enfermedad renal. Cabe mencionar que los pacientes que durante la HD presentan eventos relacionados al tratamiento perciben su calidad de vida como mala posiblemente al efecto hemodinámico brusco y/o a las hospitalizaciones que generan las infecciones. Las complicaciones más frecuentes son Hipotensión, infección de angioacceso, cefalea y crisis hipertensiva.

Recibido: 27 julio 2016

Revisado: 6 septiembre 2016

Modificado: 3 marzo 2017

Aceptado: 5 abril 2017

\section{Bibliografía}

1. Mezzano S, Aros C. Enfermedad renal crónica: clasificación, mecanismos de progresión y estrategias de renoprotección. Rev. méd. Chile. 2005, vol.133, n.3. pp. 338-348.

2. Ramírez T. y cols. Complicaciones médicas en enfermos renales crónicos que reciben hemodiálisis periódicas. Medpub Journals España Vol. 7; 2011; no.12.

3. Amato D y cols. Prevalence of chronic kidney disease in an urban Mexican population. Kidney International, vol. 68, supplement 97; (2005). Pp. S11-S17.

4. Zuñiga, C., Muller, 0. H., \& Flores, O. M. (2011). Prevalencia de enfermedad renal crónica en centros urbanos de atención primaria. Revista Médica Chile, 139, 1176 -1184.

5. Chaves K, Duarte A, Vesga J. adaptación transcultural del cuestionario KDQOL SF 36 para evaluar calidad de vida en pacientes con enfermedad renal crónica en Colombia. rev.fac. med vol.21 no.2 Bogotá July/Dec. 2013.

6. Gorriz J, y cols. Significado pronóstico de la diálisis programa en pacientes que inician tratamiento sustitutivo renal. Un estudio multicéntrico español. Nefrología; 2002(22). 
7. Loza-Concha, R. E., \& Quispe, A. M. (2011). CostoUtilidad del trasplante renal frente a la hemodiálisis en el tratamiento de la insuficiencia renal crónica terminal en un hospital peruano. Rev. Perú Med. Exp Salud Pública, 28(3), 432-439.

8. Mera M. calidad de vida de pacientes con insuficiencia Renal Crónica Terminal en tratamiento sustitutivo con hemodiálisis Centro Médico Dial Sur Osorio. (Tesis). Chile. Universidad de Austral de Chile. Facultad de Medicina; 2007.

9. Gill, T.M. \& Feinstein, A.R. (1994). A critical appraisal of the quality of quality-of-life measurements. Journal of the American Medical Association, 272 (8), 619-626.

10. Urzúa $A$, Caqueo A. Calidad de vida: una revisión teórica del concepto. Ter Psicol .2012; (30).61-71.

11. Vázquez I, Valderrábano $F$, Jofre $R$ et al. Psychosocial factors and quiality of life in youg hemodialysis patients with low comorbidity. J Nephrol 2003; 16: 886-248.

12. Reyes F, Canseco J, Vásquez S, Reyes V. Evaluación de la calidad de vida reacionada con la salud en pacientes con enfermedad renal crónica en terapia de reemplazo mediante hemodiálisis en el hospital regional de alta especialidad e Oaxaca. Evid Med Invest Salud. 2012; 5 (3): 89-94.
13. Informe sobre la salud de los mexicanos. Diagnóstico general de la salud poblacional 2015.

14. Declaración de Helsinki de la Asociación Médica Mundial. Principios éticos para las investigaciones médicas en serse vivos. Disponible en línea: http:// bit.ly/1MK2xtC

15. Zuñiga C, y cols Evaluación de la calidad de Vida en pacientes en hemodiálisis crónica mediante el cuestionario "Kidney Disease Quality of life (kdqol-36)" Rev Med Chile 2009; 137: 200-207.

16. Sánchez $A$, Zavala-Méndez $M$, Pérez-Pérez $A$. Hemodiálisis: proceso no exento de complicaciones. Rev Enferm Inst Mex Seguro Soc. 2012;20(3): $131-137$.

17. Fernández-Samos $R$, y cols. Acceso vasculares y calidad de vida en la enfermedad crónica renal terminal. Angiología 2005; 57(2): S185-S198. 